\title{
The long and the short of it: unlocking nanopore long-read RNA sequencing data with short-read tools
}

\author{
Xueyi Dong ${ }^{1,2, \bigotimes}$, Luyi Tian ${ }^{1,2}$, Quentin Gouil ${ }^{1,2}$, Hasaru Kariyawasam ${ }^{1}$, Shian $\mathrm{Su}^{1,2}$, Ricardo De Paoli-Iseppi ${ }^{3}$, Yair David \\ Joseph Prawer $^{3}$, Michael B. Clark ${ }^{3}$, Kelsey Breslin ${ }^{1}$, Megan Iminitoff ${ }^{1,2}$, Marnie E. Blewitt ${ }^{1,2}$, Charity W. Law ${ }^{1,2,1}$, and Matthew \\ E. Ritchie ${ }^{1,2, \bigotimes}$ \\ ${ }^{1}$ Epigenetics and Development Division, The Walter and Eliza Hall Institute of Medical Research, 1 G Royal Parade, Parkville, Victoria 3052, Australia
${ }^{2}$ Department of Medical Biology, The University of Melbourne, Parkville, Victoria 3010, Australia \\ ${ }^{3}$ Centre for Stem Cell Systems, Department of Anatomy and Neuroscience, The University of Melbourne, Parkville, Victoria 3010, Australia
}

\begin{abstract}
Application of Oxford Nanopore Technologies' long-read sequencing platform to transcriptomic analysis is increasing in popularity. However, such analysis can be challenging due to small library sizes and high sequence error, which decreases quantification accuracy and reduces power for statistical testing. Here, we report the analysis of two nanopore sequencing RNA-seq datasets with the goal of obtaining gene-level and isoform-level differential expression information. A dataset of synthetic, spliced, spike-in RNAs ("sequins") as well as a mouse neural stem cell dataset from samples with a null mutation of the epigenetic regulator $S m c h d 1$ were analysed using a mix of longread specific tools for preprocessing together with established short-read RNA-seq methods. We used limma-voom to perform differential gene expression analysis, and the novel FLAMES pipeline to perform isoform identification and quantification, followed by DRIMSeq and limma-diffSplice (with stageR) to perform differential transcript usage analysis. We compared results from the sequins dataset to the ground truth, and results of the mouse dataset to a previous short-read study on equivalent samples. Overall, our work shows that transcriptomic analysis of long-read nanopore data using short-read software and methods that are already in wide use can yield meaningful results.
\end{abstract}

Correspondence: mritchie@wehi.edu.au

dong.x@wehi.edu.au, law@wehi.edu.au,

\section{Introduction}

Short-read sequencing technology has underpinned transcriptomic profiling research over the past decade. The sequencing platforms offered by companies such as Illumina Inc. provide high read accuracy ( $>99.9 \%$ ) and throughput which allows many samples to be profiled in parallel. One major limitation of short-read sequencing technology is the modest read lengths offered (currently up to 600 bases), which makes accurate isoform quantification and novel isoform discovery challenging. Long-read sequencing offers a distinct advantage in this regard, with the ability to generate reads that are typically in the 1-100 kilobase (kb) range(1), which spans the typical length distribution of spliced genes in human (for protein coding genes $1-3 \mathrm{~kb}$ is typical with outliers such as Titin at more than $80 \mathrm{~kb}$ ) thereby allowing the sequencing of entire isoforms. This however comes at the expense of lower throughput and reduced accuracy compared to short-read sequencing. The two main technology platforms that dominate the field of long-read sequencing are Pacific Biosciences' Single-Molecule Real Time (SMRT) sequencing and Oxford Nanopore Technologies' (ONT) nanopore sequencing.

Previous work on long-read transcriptomic data focuses on transcript-level analysis, especially in the discovery of novel isoforms(2-4). Some long-read specific methods have been developed for this task. Reference-based methods, such as TALON(5), compares reads to existing gene and transcript models to create novel models. Reference-free methods, such as $F L A I R(6)$, maps reads to the reference genome, clusters alignments into groups and collapses them into isoforms. Differential transcript usage (DTU) is another transcript-level analysis that is of great interest(6-8). DTU analyses examine differences in the relative proportions of expressed isoforms between two conditions. The DRIMSeq(9) method performs DTU analysis on transcript-level RNA-seq counts using a Dirichlet-multinomial model. Alternatively, tools developed for differential exon usage analysis, such as the diffSplice function(10) in limma and edgeR packages have also been adapted to DTU analyses(11). Both DRIMSeq and diffSplice methods were developed for short read data. The stageR package(12) can be used to control the false discovery rate (FDR) of DTU analyses through its stage-wise method which screens potential DTU genes using gene-level $p$-values before selecting the transcripts with evidence of DTU.

Previous studies have looked at gene-level analysis of nanopore data but study design limited the methods used. Soneson et al.(13) concluded that read coverage in native RNA libraries ( $\sim 0.5$ million aligned reads per flow cell) were too low for gene-level analyses, resulting in low power and high variability. Li et al.(7) worked around this by simply using fold-changes to identify differentially expressed genes for three ONT MinION direct RNA Caenorhabditis elegans samples, however the lack of statistical testing could lead to unreliable results. Jenjaroenpun et al.(14) used $D E$ Seq2(15) to perform differential expression analysis on direct RNA transcript-level counts, but gene-level expression was not studied.

In this study, we performed gene- and isoform-level analyses of two nanopore long-read transcriptome sequencing datasets that follow a simple replicated experimental design: a synthetic "sequins"(16) PCR-cDNA dataset, and a mouse neu- 
ral stem cell direct-cDNA dataset. We obtained meaningful results using an analysis pipeline that mostly comprised of "off-the-shelf" methods developed for short-read data, despite our datasets having only a few million long reads per sample. We found our results for the common two-group experimental design to be reliable in that they are broadly consistent with the available ground-truth or findings from a previous short-read experiment. We found existing methods for isoform identification from long-read data to be unreliable, and introduce a novel method, FLAMES, as part of our isoform-level analysis pipeline.

\section{Materials and methods}

Study design. Mouse neural stem cells (NSCs) from 4 wild type (WT) and 3 MommeD1 mutated (Smchdl-null)(17) samples were prepared and sequenced, together with 3 "other" samples from a different experiment. Samples were sequenced in two batches, each containing 6 samples. One WT and one "other" sample were sequenced in both batches as technical replicates.

Technical replicates of synthetic "sequin" RNA standards(16) from two mixes (A and B) were prepared and sequenced. These samples contain the same transcripts but at variable molar ratios to simulate biological differences in gene expression and alternative splicing. Among the 76 synthetic genes, 21 were up-regulated and 23 were down-regulated in mix B compared to mix A. The corresponding transcripts of 28 genes were expressed at different proportions between the two mixes, resulting in DTU for 62 out of 160 transcripts.

Biological materials. Synthetic "sequin" RNA standards were obtained from the Garvan Institute of Medical Research.

NSCs were derived as described in Chen et al.(18). Cells were grown in NeuroCult Stem Cell medium (StemCell Technologies \#05702) with cytokines: NeuroCult NSC Basal Medium (Mouse) (StemCell Technologies \#05700) supplemented with NeuroCult Proliferation Supplement (Mouse) (StemCell Technologies \#05701), $0.25 \mathrm{mg} / \mathrm{mL}$ rh EGF (StemCell Technologies \#02633) and $0.25 \mathrm{mg} / \mathrm{mL}$ rh bFGF (StemCell Technologies \#02634). We extracted total RNA with Trizol and purified polyA RNA with the NEBNext Poly(A) mRNA Magnetic Isolation Module (E7490).

Nanopore sequencing and data preprocessing. Sequin cDNA libraries were constructed with SQK-PCS109 cDNAPCR sequencing and SQK-PBK004 PCR Barcoding kits using the supplied protocol. Briefly, duplicate libraries of each mix (A1, A2, B1, and B2) were constructed using $15 \mathrm{ng}$ as input for cDNA synthesis. Samples were barcoded 1 to 4 using the supplied PCR barcodes. Transcripts were amplified by 14 cycles of PCR with a 6-minute extension time.

Sequencing libraries were individually purified using Beckman Coulter 0.8x AMPure XP beads and quantified using an Invitrogen Qubit 4.0 Fluorometer (ThermoFisher Scientific). Equimolar amounts of each sample were pooled to a total of approximately $160 \mathrm{fmol}$ (assuming median transcript size is $1 \mathrm{~kb}$ ), and quality control of the pooled library was performed using Agilent Technologies TapeStation 4200. The final library was loaded onto an R9.4.1 MinION flow cell and sequenced for 65 hours with a buffer refuel at 24 hours (using $250 \mathrm{~mL}$ buffer FB) using the ONT GridION platform. The fast 5 files were base-called by Guppy version 3.2.8 using configuration file dna_r9.4.1_450bps_hac.cfg to obtain fastq files. MinKNOW version 3.6.0 was used to trim adaptor sequences and demultiplex barcoded reads. Both Guppy and MinKNOW are only available to ONT customers via the community site (https://community. nanoporetech.com/).

For the NSC dataset we prepared direct-cDNA libraries from $40 \mathrm{ng}$ purified polyadenylated RNA. We combined the ONT direct-cDNA sequencing (SQK-DCS108) protocol (version DCB_9036_v108_revG_30Jun2017) with the one-pot native barcoding protocol(19) with extended incubation times (using SQK-LSK109 and EXP-NBD103 kits) for library preparation of the first batch, and used the updated kits SQK-DCS109 and EXP-NBD114 for the second batch (protocol PDCB_9093_v109_revA_04Feb2019). We loaded $100 \mathrm{ng}$ of the final libraries on one PromethION flow cell (FLO-PRO002) per batch. The fast 5 files were base-called by Guppy version 3.1.5 using configuration file dna_r9.4.1_450bps_hac_prom.cfg to yield fastq files. We used Porechop (20) to trim adaptor sequences from reads and demultiplex barcoded reads. The "other" samples were removed in downstream analysis. For an overview of our analysis pipeline see Figure 1A.

Genomic alignment. The NSC reads were aligned to mouse mm10 genome using minimap2 version 2.17-r943-dirty(21) to get bam files. The genome alignments were performed with the arguments

-ax splice -uf -k14 -junc-bed, allowing spliced alignments on the forward transcript strand to map with higher sensitivity. It also uses annotated splice junctions to improve the accuracy of mapping at junctions. Gencode release M23 (GRCm38.p6) annotation(22) was used to provide information on known splicing junctions. The sequins ONT reads were mapped to the artificial chromosome chrIS_R using minimap 2 with the arguments -ax splice $-M D$. The bam files were sorted and indexed using samtools version 1.6(23).

Gene abundance estimation. Mapped reads were assigned to individual genes and counted by the featureCounts(24) function in the $\mathrm{R}(25) /$ Bioconductor(26) package Rsubread version 1.34.4 $(27,28)$. We used the in-built $\mathrm{mm} 10$ annotation for the NSC data, and sequins annotation GTF file version 2.4 for the sequins data. Arguments isLongRead $=$ TRUE and primaryOnly=TRUE were used to indicate that the input data contains long reads and to count primary alignments only.

Differential gene expression analysis. Genes in the NSC dataset were annotated using R/Bioconductor package Mus.musculus(29), and read counts from technical repli- 
bioRxiv preprint doi: https://doi.org/10.1101/2020.06.28.176727; this version posted June 29, 2020. The copyright holder for this preprint (which was not certified by peer review) is the author/funder, who has granted bioRxiv a license to display the preprint in perpetuity. It is made available under aCC-BY-ND 4.0 International license.
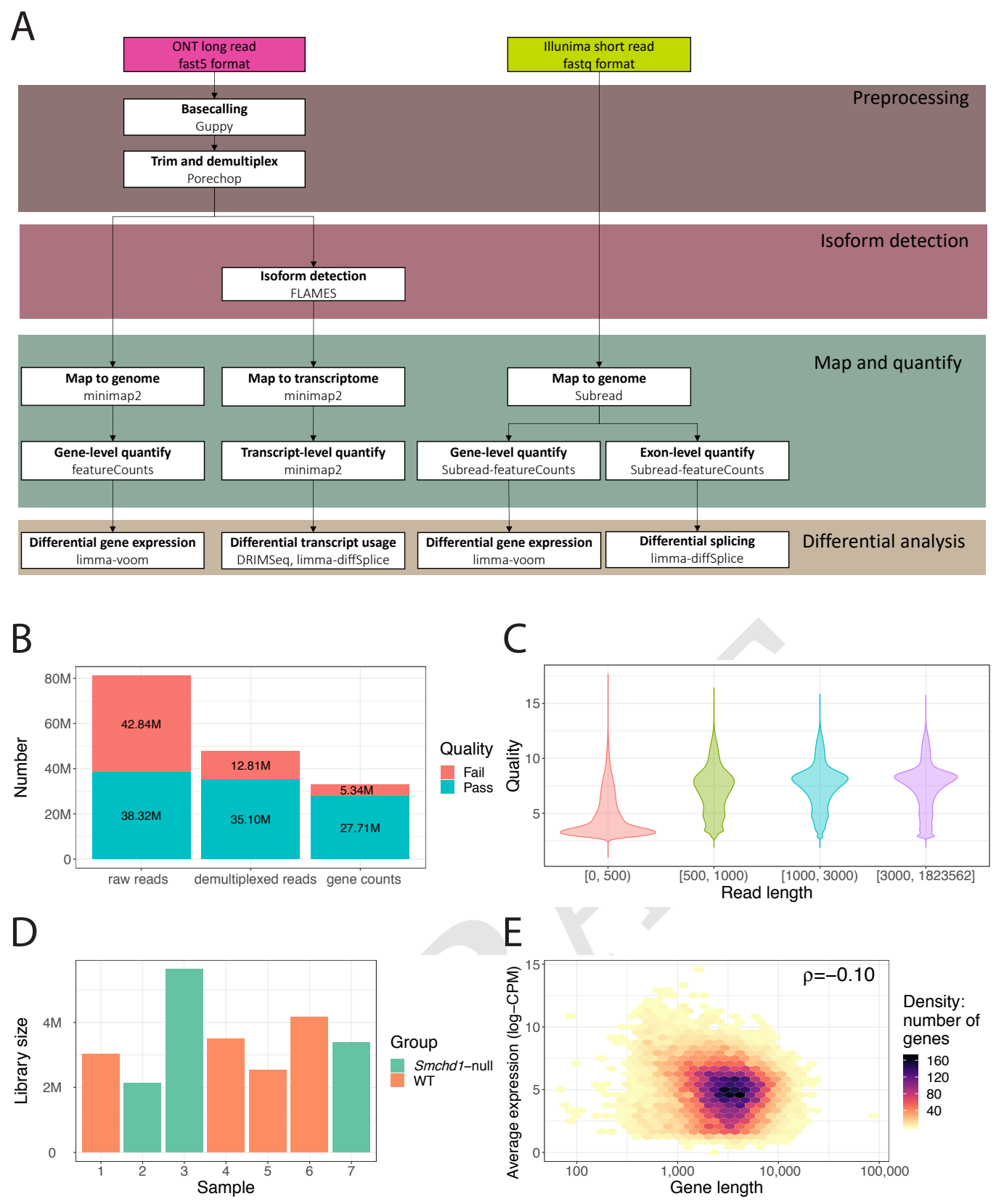

Fig. 1. Analysis workflow and quality metrics. (A) Overview of the analysis workflow used to process the mouse NSC direct-cDNA long-read and short-read RNA-seq data. (B) The number of raw reads, trimmed and demultiplexed reads and gene-level counts in the NSC dataset, stratified by average base quality score (Pass: >7, turquoise; Fail: $\leq 7$, red). (C) Distribution of read quality in the NSC dataset, stratified by read length. Read quality is defined by the average base quality score of a read. (D) The total number of reads assigned to each sample in the NSC dataset (green: Smchd1-null samples; orange: WT samples). (E) A hexagonal 2D density plot showing the correlation between gene length and average gene expression (log-CPM) in the NSC dataset.

cates were combined. For both datasets, we organized and preprocessed the count data using the R/Bioconductor package edgeR version 3.26.8(30, 31). Lowly expressed genes were removed using the filterByExpr function with default arguments. Normalization factors were calculated using the trimmed mean of M-values method(32). Differential gene expression (DGE) analysis was performed using the limma-voom pipeline version $3.40 .6(10,33,34)$, with sample-specific quality weights(35). Linear models were fitted with either genotype or sequin mix information to create the design matrix, followed by empirical Bayes moderation of $t$-statistics(36). Raw $p$-values were adjusted for multiple testing(37).

Mouse NSC short-read data. We obtained DGE results from a previous Illumina short-read RNA-seq study comparing mouse Smchdl-null and WT NSC samples $(18,38)$ available at http://bioinf.wehi.edu.au/folders/ smchd1/ and from GEO (accession number GSE65747). Using a limma-voom pipeline, the study reported 1,197 differentially expressed (DE) genes (adjusted $p$-value cutoff of 0.01 ). We further restricted this list (adjusted $p$-value cutoff of 0.0001) to give us 218 up- and 54 down-regulated genes in Smchdl-null samples when compared to WT samples. This 
cutoff resulted in similar numbers of significant genes between the short- and long-read datasets. The DE genes were compared to that of the NSC long-read data using ROAST gene set testing(39) with 9,999 rotations.

Transcript-level analysis. We used three different tools to perform isoform detection and quantification: FLAIR version 1.5.0(6) and TALON version 5.0.0(5) for the sequins data, and FLAMES version 0.1.0 for both sequins and NSC datasets. Default parameters were used to run FLAIR. TranscriptClean(40) version 1.02 which performs referencebased error correction was applied prior to running TALON version 5.0. Transcripts identified by TALON were filtered using default setting.

FLAMES (short for 'Full-Length trAnscript quantification, Mutation and Splicing analysis') is a novel method and software tool developed for long-read RNA-seq data, available at https://github.com/LuyiTian/FLAMES. It requires sorted bam files with reads aligned to the genome as input. Scanning each gene, FLAMES groups reads that are similar as isoforms (transcript start/end site differs by $<100 \mathrm{bp}$ and splice site differs by $<10 \mathrm{bp}$ ). The isoforms are then compared against a reference annotation (Gencode release M23 (GRCm38.p6)(22)), and transcript sequences are extracted from the isoform assembly. All reads are then realigned to both the known and assembled transcripts and quantified. High-confidence isoforms are those identified with at least 10 supporting reads.

After running each isoform detection tool, SQANTI(41) was used to classify identified isoforms by comparing them to the annotation. Lowly expressed transcripts were removed from downstream analysis. We kept transcripts with 10 or more counts in at least 3 samples in the NSC data, and in at least 2 samples in the sequins data. For both datasets, genes in every sample were also required to have an associated gene count (obtained by summing counts across all transcripts for a given gene) of 10 or more.

DTU analysis was performed using two methods: the R/Bioconductor package DRIMSeq version 1.12.0(9), and diffSplice from the limma package version 3.40.6. Originally, DRIMSeq was designed for use with transcript-level counts in short-read data, giving adjusted $p$-values at both the gene-level and feature-level (transcripts). DiffSplice analyses exon-level counts in short-read data to indirectly call for differences in isoform proportions, and reports adjusted $p$ values at the gene-level (Simes adjustment and/or F-tests) and exon-level ( $t$-tests). For long-read data, we applied the diffSplice to transcript-level counts rather than exon-level counts as carried out by Love et al.(11). Additionally, the stagewise method from R/Bioconductor package stageR version 1.6.0(12) was also applied to the raw $p$-values from DRIMSeq (gene- and transcript-level) and diffSplice (Simes and $t$-tests) methods for FDR control to give stageR gene- and transcriptlevel adjusted $p$-values.

Data and code availability. RNA-seq data can be accessed from Gene Expression Omnibus (GEO) under accession numbers GSE151984 (sequins) and GSE151841 (NSC data).
All code used to perform these analyses are available from https://github.com/XueyiDong/LongReadRNA.

\section{Results}

Data quality. To assess the quality of our long-read datasets, raw long reads were pre-processed and assigned to gene-level counts using an appropriate reference genome. Figure 1B shows the number of reads (or amount of information) retained after some crucial steps in processing the NSC data. A total of $\sim 81$ million reads were successfully sequenced and base-called. A relatively small proportion of those, $\sim 60 \%$ or $\sim 48$ million reads, were detected with adaptor and barcode sequences in the trimming and demultiplexing steps. Most of the reads that did not pass these steps were of low quality and marked as "fail" reads (average base quality score $\leq 7$ ). The reads were then mapped to the genome and assigned to genes, producing $\sim 28$ million gene-level counts. For the sequins dataset, $\sim 7.3$ million raw "pass" reads yielded $\sim 5.7$ million gene-level counts (Supplementary Figure S1).

In the NSC dataset, median read length is about 800 bases, where low quality "fail" reads are generally shorter (median of $\sim 300$ bases). Figure 1C shows that short reads $(<500$ bases) tend to have low read quality relative to longer reads, similar to that observed in Soneson et al.(13). The quality of "extra long" reads $(\geq 3000$ bases) were similar to that of "long" (1000 to 2999 bases) and "medium" (500 to 999 bases) length categories, indicating Nanopore's ability to detect transcripts in this size range. A small proportion $(<1 \%)$ of reads exceed 5 kilobases. Similar to the NSC dataset, the sequins dataset had a median read length of 996 bases, which is sightly longer than its expected value of 908 bases.

The library size (sum of gene counts) of samples in the NSC dataset varied between 2.1 million to 5.6 million reads (Figure 1D). As comparison, the library size of samples in the short-read NSC study(18) were ranging between 18.6 million to 23.2 million reads.

Gene expression analysis. In short-read RNA-seq, transcripts (or genes) are fragmented for sequencing, such that longer transcripts can be over-represented relative to transcripts that are shorter. As a consequence, DGE analyses are biased towards the detection of genes (or transcripts) that are relatively long(42). Also, DGE analyses may be confounded by DTU, such that gene-level counts are affected by the varying proportions of transcripts with varying lengths. One advantage to long-read RNA-seq protocols is that they do not include the fragmentation step, and should theoretically be unbiased to gene length. To examine this, we looked at the relationship between gene length and gene expression using $\log _{2}$-counts-per-million $(\log$-CPM) values. Gene length is weakly associated with expression in both long-read datasets; Pearson correlation of 0.10 for the NSC dataset (Figure 1E), and -0.05 for sequins dataset (Supplementary Figure S2). Whereas, correlation in the short-read NSC study(18) is greater, at 0.20 .

We applied the limma-voom workflow designed for shortread DGE analysis to our long-read data. We first anal- 


\section{A}

B
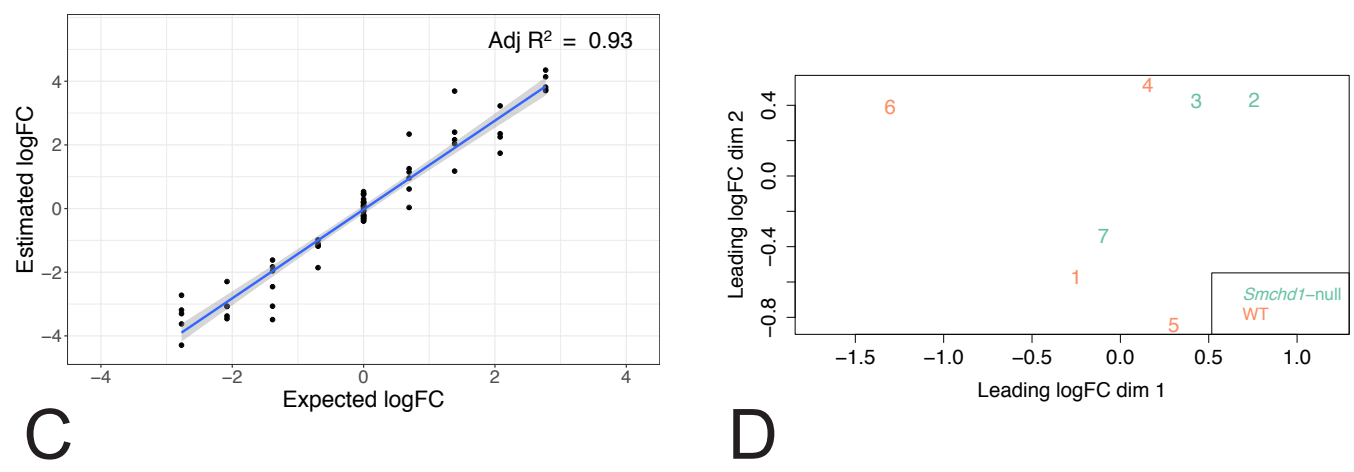

D
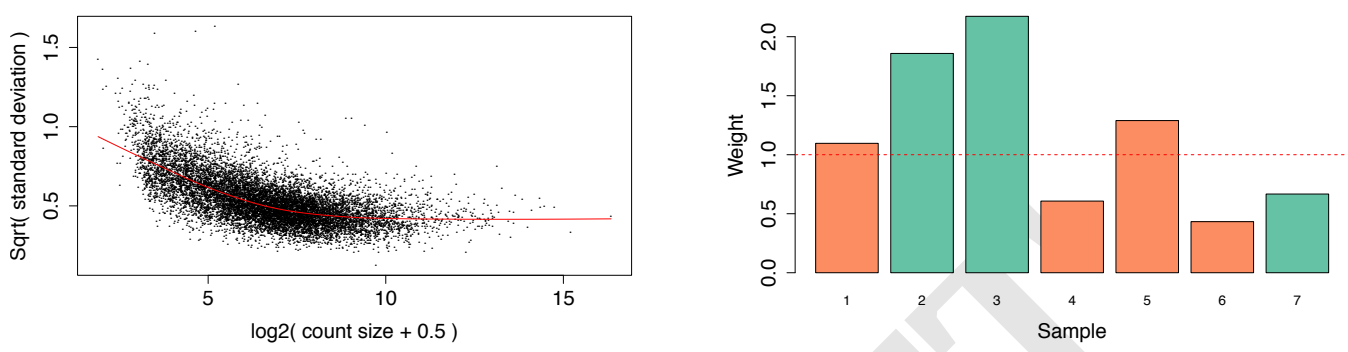

$\mathrm{E}$
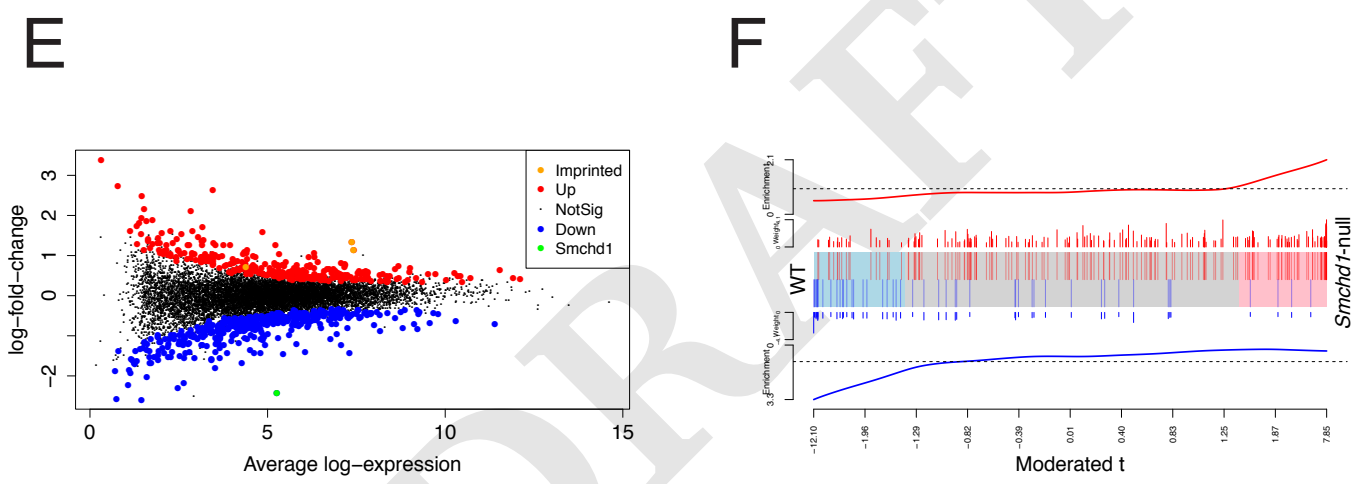

Fig. 2. Results for differential gene expression analysis. (A) Correlation between the observed $\log _{2} F C$ between mix $A$ and $B$ and the expected log $F C$ in the sequins data. The blue line is the linear regression line. (B) MDS plot showing the relationship between NSC samples based on gene-level logCPM. (C) Voom mean-variance trend in NSC data where points represent genes, and (D) sample-specific weights obtained from the limma-voom workflow (colour-coded by genotype). (E) Gene-level plot of logFC for Smchdl-null versus WT plotted against average $\log _{2}$-expression values. Differentially expressed genes are highlighted (red: up-regulated genes, blue: down-regulated genes). (F) The barcode plot shows the correlation between our long-read differential expression results and the results from a previous short-read dataset collected on the same NSC sample types. Each vertical bar represents a DE gene from the previous short-read study (red: up-regulated genes, blue: down-regulated genes), and the position of the bar on the x-axis represents the moderated t-statistic of the same gene in our long-read results. The length of the vertical lines represent the logFC of the gene in the short-read results. The red worm on the top and the blue worm at the bottom represent the relative enrichment of the vertical bars in each part of the plot.

ysed the sequins data to check whether the approach was appropriate using the ground-truth available. The analysis was carried out using voomWithQualityWeights to account for sample-level heterogeneity by estimating sample-specific quality weights based on similarity of gene expression within the same group. The sample weights were combined with voom precision weights that are based on the mean-variance relationship estimated from the data. Even though there were only 69 genes present in the dataset (as opposed to tens of thousands in a typical dataset), the mean-variance trend observed for the sequins data (Supplementary Figure S3) was similar to that of short-read RNA-seq data(33).

Linear modelling on the gene-level counts were carried to obtain estimated $\log _{2}$ fold-change $(\log \mathrm{FC})$ values between mix
$A$ and B. Estimated values were highly correlated $\left(R^{2}=0.933\right)$ with expected $\log \mathrm{FCs}$ (Figure 2A). Using an adjusted $p$-value cutoff of $0.05,21$ down-regulated and 18 up-regulated genes were detected between mix B and A. There were no false discoveries, and only 2 of the truly differentially expressed genes were not detected. Overall, results from the sequin synthetic control data indicate that the limma-voom pipeline is powerful and reliable when applied to long-read data, so we applied it to the NSC dataset also.

Unsupervised clustering by multidimensional scaling (MDS) was used to observe the relationships between NSC samples. Dimension 1 in the MDS plot roughly separates samples by genotype (Figure 2B), although a Smchdl-null sample (sample 7) is positioned more closely to WT samples. 
bioRxiv preprint doi: https://doi.org/10.1101/2020.06.28.176727; this version posted June 29, 2020. The copyright holder for this preprint (which was not certified by peer review) is the author/funder, who has granted bioRxiv a license to display the preprint in perpetuity. It is made available under aCC-BY-ND 4.0 International license.

A

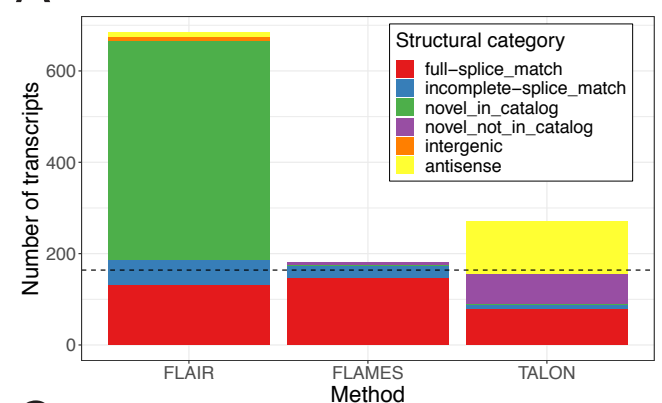

C

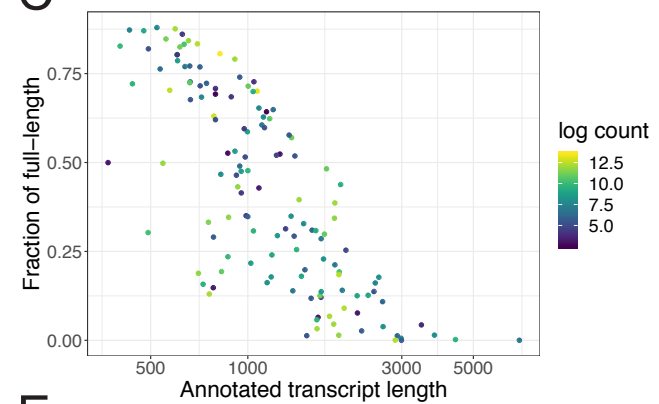

E
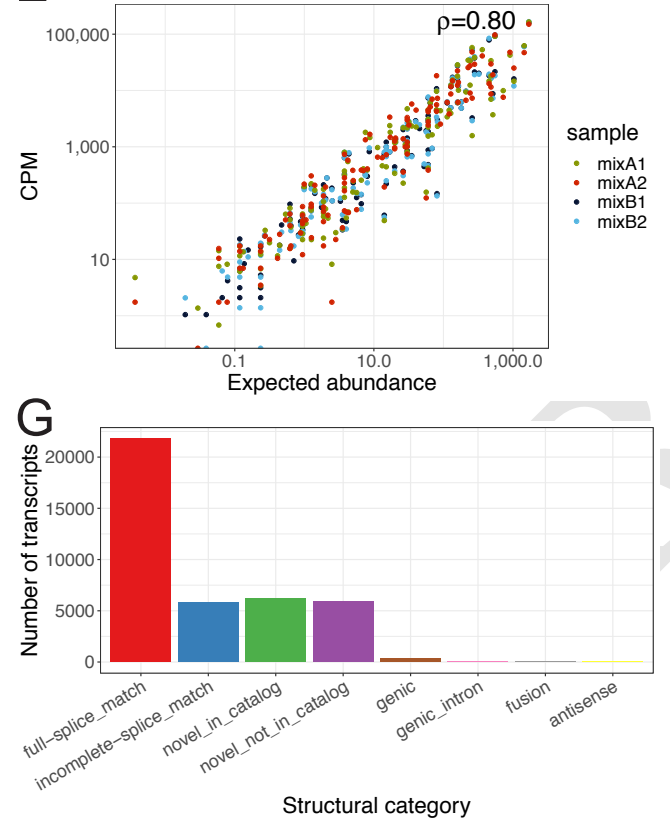

B

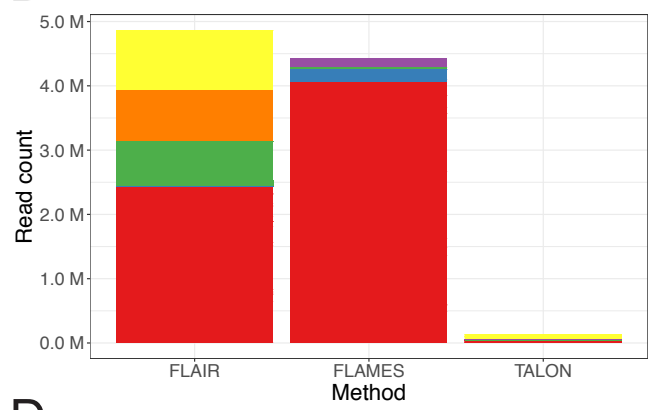

D

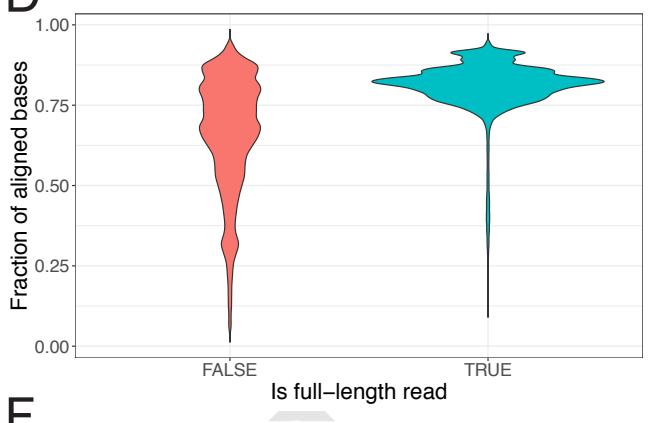

F

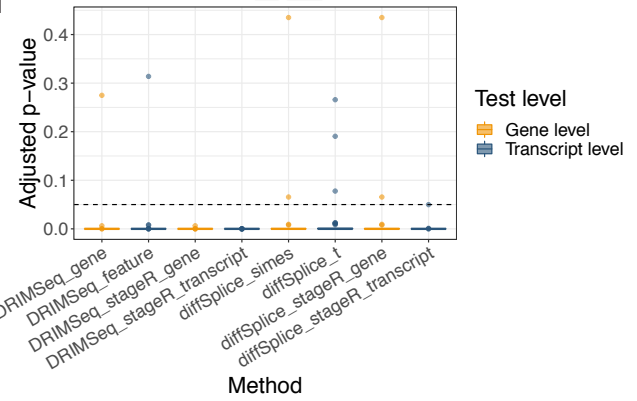

$\mathrm{H}$

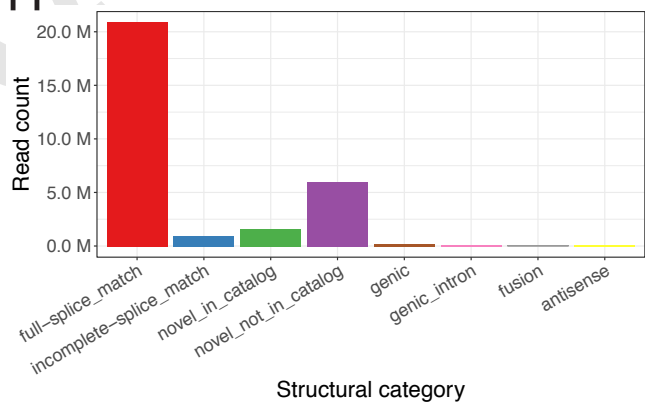

Fig. 3. Isoform identification and differential transcript usage analysis. (A) A bar plot showing the number of discovered isoform types in the sequins dataset. The bars are separated into isoform categories (by colour), and the dashed line represents the true number of isoform types. (B) A bar plot showing the number of counts from isoforms in the sequins dataset. The bars are separated into isoform categories (by colour) from which the counts are associated with. (C) A scatter plot showing the correlation between the fraction of full-length reads assigned to a transcript and the length of the annotated transcript. Dots are coloured by log ${ }_{2}$ count of transcripts. (D) A violin plot showing the aligned fraction of reads (calculated as the aligned length divided by read length), stratified by whether the read is full-length. (E) The correlation between observed transcript counts and expected transcript abundance of each gene from each sequins sample. (F) A box plot showing the distribution of adjusted $p$-values from different tests of DTU for the true DTU genes or transcripts in sequins data. The dashed horizontal line shows the adjusted $p$-value cutoff of 0.05 . Boxes are coloured by whether the test is performed on gene level or transcript level. (G) A barplot showing the number of discovered isoforms in each category output by FLAMES in the NSC dataset. (H) A barplot showing the number of counts from isoforms in each category output by FLAMES in the NSC dataset.

The mean-variance trend for this dataset is strikingly similar to that which is typically observed in short-read RNAseq experiments(33) (Figure 2C); even more so than in the sequins dataset. Estimated sample weights favoured samples that distinguished groups across dimension 2 of the MDS plot, giving samples 2 and 3 in the Smchd1-null group weights that are greater than 1 , as well as samples 1 and 5 in the WT group (Figure 2D). Using the default adjusted $p$ value cutoff of 0.05 , only 17 genes were detected as DE. Using a more liberal adjusted $p$-value cutoff of 0.25 to account for the small library sizes, detected 413 down-regulated and 321 up-regulated genes between Smchdl-null and WT samples (Figure 2E). The Smchdl gene, which was depleted in Smchdl-null samples, was detected as the most significantly 
down-regulated gene in the comparison (highlighted in Figure 2E) and serves as a positive control for our analysis.

In a previous short-read study on the same mouse NSC groups $(18,38)$, the imprinted genes Ndn, Mkrn3 and Peg12 were reported as up-regulated. These genes were also found to be $\mathrm{DE}$ in the long-read dataset (highlighted in Figure 2E). Further comparison between the short- and long-read datasets was carried out using a barcode plot (Figure 2F). The barcode plot shows that most of the genes that were up-regulated in the short-read dataset (red vertical lines in the plot) also tend to be up-regulated in our long-read dataset (positioned towards the right of the plot). Specifically, the genes that were most highly up-regulated in the short-read dataset as ranked by $\log \mathrm{FC}$ (long red vertical lines), are also highly upregulated in the long-read data (further right in the plot). The same goes for down-regulated genes in the short-read dataset (blue vertical lines in the plot), which tend to be downregulated in the long-read dataset (positioned towards the left of the plot). We tested concordance of the datasets formally by applying the ROAST gene set testing method(39) to our long-read data. Using both up- and down-regulated gene sets from the short-read dataset, weighted on their $\operatorname{logFC}$ values, ROAST returned an "up" $p$-value of 0.10 , which indicates that transcriptional changes for the comparison of Smchdlnull versus WT are somewhat consistent between the two datasets (up-regulated genes in the short-read data tend to be up-regulated in the long-read data, and down-regulated genes in the long-read data tend to be down-regulated in the longread data). The relatively large ROAST $p$-value and overall lack of power to detect differentially expressed genes is likely due to relatively low sequencing levels per sample and within-genotype sample heterogeneity in the long-read experiment.

Transcript-level analysis. Transcript-level analysis of nanopore RNA-seq data usually starts with isoform detection. To test which tool is best suited to nanopore data, we compared two popular tools, FLAIR and TALON with our novel FLAMES pipeline on the sequins dataset. Ideally, all transcripts that appear in the sequins annotation should be detected, and there should not be any novel isoforms. Our results showed that FLAMES detected the most sequin transcripts (Figure 3A, 'full splice match' category) and the fewest artefactual isoforms (Figure 3A, other categories). While most sequin transcripts were also detected by FLAIR, a disproportionately large number of artefactual isoforms were also identified, especially those classified as 'novel in cata$\log$ ' for which we know there are none. TALON only detected about half of the sequin transcripts, and many antisense isoforms. When we looked into the number of reads assigned to transcripts in each category (Figure 3B), the majority of counts in FLAMES were from known isoforms, while about half of the counts in FLAIR were from artefactual isoforms. The total number of read counts from FLAMES $(\sim 4 \mathrm{M})$ and FLAIR $(\sim 2.4 \mathrm{M})$ are similar, while for TALON it was much lower $(\sim 4.4 \mathrm{~K})$. Results from the sequins dataset indicated that the novel FLAMES pipeline outperformed the other two methods.
To further assess the performance of FLAMES and the quality of the dataset, we calculated the coverage fraction of transcripts by individual reads. Here, reads covering $95 \%$ or more of the bases of their corresponding transcript are defined as "full-length". In our sequins data, $48 \%$ of reads were found to be full-length. Reads assigned to longer transcripts are less likely to be full-length (Figure 3C), consistent with findings from Jenjaroenpun et al.(14). We also observed that full-length reads have a higher fraction of aligned bases (Figure 3D). Looking further into the isoforms identified by FLAMES, we found that 'full splice match' isoforms tend to be longer than those in the other categories (Supplementary Figure S4), but they tend to miss some bases at the transcription start sites and/or transcription termination sites. This suggests that some reads may be truncated in our sequins dataset, which presumably occurs during library preparation of sequencing.

Next, we adapted DRIMSeq and the diffSplice function in limma for DTU analysis of long-read data. We also combined the methods with the stage-wise analysis from the stageR(12) package since it was recommended in the DRIM$\mathrm{Seq}$ vignette for statistical improvement and enhanced biological interpretation of results. We expect good performance of DTU analyses comparing mixes A and B since the observed CPM values of sequin transcripts were highly correlated with their expected abundances (Figure 3E). Indeed, all of the DTU methods performed well, with no false discoveries for any of the methods (using an adjusted $p$-value cutoff of 0.05) (Figure 3F, Supplementary Figure S5). The true positive rate (TPR) (detecting genes/transcripts as having DTU when they truly have DTU) was very high for all of the methods (TPR $>0.89$ ), with DRIMSeq slightly outperforming diffSplice (Supplementary Figure S6). StageR transcript-level testing further improved the results of both DRIMSeq and diffSplice (TPR=1). StageR gene-level testing also improved the results from DRIMSeq, but not for diffSplice. We demonstrate that our pipeline and combination of methods for transcript-level analysis produces accurate transcript quantification and identification of DTU, and provides confidence for application to other long-read transcriptomic datasets.

We then applied our transcript-level analysis workflow to the NSC dataset. The FLAMES pipeline returned 40,221 unique isoforms from 10,986 genes, of which $48 \%$ were classified as novel (Figure $3 \mathrm{G}$ ), which is a lot more than what was observed in the sequins dataset. Since we observed a very low level of falsely discovered isoforms in the sequins data, we assume that that most of these novel isoforms are real. This suggests that the current mouse transcript annotation is incomplete. Of the mapped reads, $29.4 \%$ were assigned to novel isoforms, the majority of which were from the 'novel not in catalog' category (Figure 3H). Both DTU methods (DRIMSeq and diffSplice) found only one gene Pisd as having DTU for Smchdl-null versus WT using an adjusted $p$ value cutoff of 0.25 . Pisd was not DE at the gene-level (adjusted $p$-value $=0.44$ ). Transcript ENSMUST00000201980.4 in Pisd was identified to have differential usage between the 
two groups (Supplementary Figure S7 and S8).

Using limma-diffSplice applied to exon counts, we also performed differential exon usage analysis on the NSC shortread data and found 87 significant genes (adjusted $p$-value cutoff of 0.05). The results, however, were discordant between short- and long-read datasets, with only 3 common genes in the top 200 most significant genes identified by limma-diffSplice for both datasets. Pisd was not identified to have significant differential splicing in the short-read analysis (adjusted $p$-value $=1.00$ ).

\section{Discussion}

Our DGE analysis uses a limma-voom workflow and shows that results from PCR-cDNA and direct-cDNA long-reads are reliable, such that estimated results are comparable to the known truth in the sequins synthetic control dataset, and concordant with that of a previous short-read study for the NSCs. Although the total library size in the sequins dataset is lower than that of the NSC dataset, more reads were assigned per gene since the dataset contains a small set of genes, which improved power for DGE analysis. Overall, comparisons using long-read experiments suffer from a lack of statistical power due to low library sizes. It would be desirable for longread transcriptomic studies to have total read numbers that are more comparable to what is routinely achieved in shortread experiments (20-50M reads per sample is not unusual). We expect this to occur in the near future as throughput of long-read experiments increases.

We also looked into transcript-level analysis of long-read data and found our novel FLAMES pipeline to be reliable in both isoform detection and quantification. The high false positive rate of FLAIR for isoform detection suggests that its reference-free algorithm needs further improvement to adapt to high error rates in long-read sequencing. Despite methods being designed originally for short-read data, DRIMSeq and diffSplice (in combination with stageR or not) performed very well in DTU analyses of our sequins data using transcriptlevel counts. We believe these methods could be applied to other datasets with confidence, but may lack power to detect DTU genes if transcript counts are very low. A potential issue in the application of the methods to our NSC dataset is that altered expression of the gene (Smchdl) may not affect RNA splicing mechanisms. Discordance between our results for short- and long-read NSC studies may be a reflection of the differences in exon-level counting in short reads versus transcript-level counting in long reads, rather than inconsistencies in the diffSplice method itself. Notably, relative to DGE analyses, a DTU analysis further splits gene-level counts into associated isoforms which reduces power for statistical testing. For this reason, the power to detect DTU genes in the NSC long-read dataset is reduced relative to the sequins dataset since the latter contains far fewer expressed genes and transcripts to begin with, such that transcripts have higher counts on average.

Our study is the first to test a pipeline for gene-level DGE analysis and transcript-level DTU analysis of nanopore longread RNA-seq data. Whilst the sequencing depth is relatively low, we are still able to obtain reasonable results using preexisting methods designed for short reads, namely the limma and DRIMSeq software. We expect that other short-read tools such as $e d g e R$ and DESeq 2 may also be appropriate, although this has not been tested. Exploring the strengths and weaknesses of different analysis methods on data arising from both the Nanopore and PacBio long-read platforms using a specially designed benchmarking dataset is planned as future work.

We hope that our analysis will encourage further research into the potential for long-read RNA-seq to be used in place of short-read RNA-seq, allowing for the simultaneous exploration of gene-level and isoform-level changes within the same experiment in a more comprehensive way.

\section{ACKNOWLEDGEMENTS}

This project was supported by National Health and Medical Research Council (NHMRC) Project Grant (GNT1098290 to MEB and MER), a Career Development Fellowship (GNT1104924 to MER) an Early Career Fellowship (GNT1072662 to MBC), a Bellberry-Viertel Senior Medical Research Fellowship (to MEB), a Melbourne Research Scholarship to XD and LT and Victorian State Government Operational Infrastructure Support and Australian Government NHMRC IRIISS.

\section{Bibliography}

1. Martin O. Pollard, Deepti Gurdasani, Alexander J. Mentzer, Tarryn Porter, and Manjinder S. Sandhu. Long reads: their purpose and place. Human Molecular Genetics, 27(R2):R234R241, 2018. ISSN 14602083. doi: 10.1093/hmg/ddy177.

2. Ishaan Gupta, Paul G Collier, Bettina Haase, Ahmed Mahfouz, Anoushka Joglekar, Taylor Floyd, Frank Koopmans, Ben Barres, August B Smit, Steven A Sloan, Wenjie Luo, Olivier Fedrigo, M Elizabeth Ross, and Hagen U Tilgner. Single-cell isoform RNA sequencing characterizes isoforms in thousands of cerebellar cells. Nature Biotechnology, 36(12):11971202, 2018. ISSN 1087-0156. doi: 10.1038/nbt.4259.

3. Kristoffer Sahlin and Paul Medvedev. De novo clustering of long-read transcriptome data using a greedy, quality-value based algorithm. bioRxiv, 463463, 2018. doi: 10.1101/463463.

4. K. F. Au, V. Sebastiano, P. T. Afshar, J. D. Durruthy, L. Lee, B. A. Williams, H. van Bakel, E. E. Schadt, R. A. Reijo-Pera, J. G. Underwood, and W. H. Wong. Characterization of the human ESC transcriptome by hybrid sequencing. Proceedings of the National Academy of Sciences, 110(50):E4821-E4830, 2013. ISSN 0027-8424. doi: 10.1073/pnas.1320101110.

5. Dana Wyman, Gabriela Balderrama-Gutierrez, Fairlie Reese, Shan Jiang, Sorena Rahmanian, Weihua Zeng, Brian Williams, Diane Trout, Whitney England, Sophie Chu, Robert C. Spitale, Andrea Tenner, Barbara Wold, and Ali Mortazavi. A technology-agnostic long-read analysis pipeline for transcriptome discovery and quantification. bioRxiv, 672931, 2019. doi: $10.1101 / 672931$.

6. Alison D. Tang, Cameron M. Soulette, Marijke J. van Baren, Kevyn Hart, Eva HrabetaRobinson, Catherine J. Wu, and Angela N. Brooks. Full-length transcript characterization of SF3B1 mutation in chronic lymphocytic leukemia reveals downregulation of retained introns. Nature Communications, 11(1):1-12, dec 2020. ISSN 20411723. doi: 10.1038/s41467-020-15171-6.

7. Runsheng Li, Xiaoliang Ren, Qiutao Ding, Yu Bi, Dongying Xie, and Zhongying Zhao. Direct full-length RNA sequencing reveals unexpected transcriptome complexity during Caenorhabditis elegans development. Genome Research, 30(2):287-298, 2020. doi: 10.1101/gr.251512.119.3.

8. Ashley Byrne, Anna E. Beaudin, Hugh E. Olsen, Miten Jain, Charles Cole, Theron Palmer, Rebecca M. DuBois, E. Camilla Forsberg, Mark Akeson, and Christopher Vollmers. Nanopore long-read RNAseq reveals widespread transcriptional variation among the surface receptors of individual B cells. Nature Communications, 8(May):1-11, 2017. ISSN 20411723. doi: 10.1038/ncomms16027.

9. Malgorzata Nowicka and Mark D Robinson. DRIMSeq: a Dirichlet-multinomial framework for multivariate count outcomes in genomics. F1000Research, 5:1356, 2016. ISSN 20461402. doi: $10.12688 /$ f1000research. 8900.1 .

10. Matthew E. Ritchie, Belinda Phipson, Di Wu, Yifang Hu, Charity W. Law, Wei Shi, and Gordon K. Smyth. Limma powers differential expression analyses for RNA-sequencing and microarray studies. Nucleic Acids Research, 43(7):e47, 2015. ISSN 13624962. doi: 10. 1093/nar/gkv007.

11. Michael I Love, Charlotte Soneson, and Rob Patro. Swimming downstream: statistical analysis of differential transcript usage following Salmon quantification. F1000Research, 7: 952, 2018. ISSN 2046-1402. doi: 10.12688/f1000research.15398.3.

12. Koen Van den Berge, Charlotte Soneson, Mark D. Robinson, and Lieven Clement. stageR: A general stage-wise method for controlling the gene-level false discovery rate in differential expression and differential transcript usage. Genome Biology, 18(1):151, 2017. ISSN 1474760X. doi: 10.1186/s13059-017-1277-0.

13. Charlotte Soneson, Yao Yao, Anna Bratus-Neuenschwander, Andrea Patrignani, Mark D. Robinson, and Shobbir Hussain. A comprehensive examination of Nanopore native RNA sequencing for characterization of complex transcriptomes. Nature Communications, 10(1): 3359, 2019. ISSN 2041-1723. doi: 10.1038/s41467-019-11272-z.

14. Piroon Jenjaroenpun, Thidathip Wongsurawat, Rui Pereira, Preecha Patumcharoenpol, David W. Ussery, Jens Nielsen, and Intawat Nookaew. Complete genomic and transcrip- 
bioRxiv preprint doi: https://doi.org/10.1101/2020.06.28.176727; this version posted June 29, 2020. The copyright holder for this preprint (which was not certified by peer review) is the author/funder, who has granted bioRxiv a license to display the preprint in perpetuity. It is made available under aCC-BY-ND 4.0 International license.

tional landscape analysis using third-generation sequencing: A case study of Saccharomyces cerevisiae CEN.PK113-7D. Nucleic Acids Research, 46(7):e38, 2018. ISSN 13624962. doi: 10.1093/nar/gky014.

15. Michael I. Love, Wolfgang Huber, and Simon Anders. Moderated estimation of fold change and dispersion for rna-seq data with deseq2. Genome Biology, 15(12):550, 2014. ISSN 1474-760X. doi: 10.1186/s13059-014-0550-8.

16. Simon A Hardwick, Wendy Y Chen, Ted Wong, Ira W Deveson, James Blackburn, Stacey B Andersen, Lars K Nielsen, John S Mattick, and Tim R Mercer. Spliced synthetic genes as internal controls in RNA sequencing experiments. Nature Methods, 13(9):792-8, 2016. ISSN 1548-7105. doi: 10.1038/nmeth.3958.

17. Marnie E. Blewitt, Anne Valerie Gendrel, Zhenyi Pang, Duncan B. Sparrow, Nadia Whitelaw, Jeffrey M. Craig, Anwyn Apedaile, Douglas J. Hilton, Sally L. Dunwoodie, Neil Brockdorff, Graham F. Kay, and Emma Whitelaw. SmcHD1, containing a structural-maintenance-ofchromosomes hinge domain, has a critical role in X inactivation. Nature Genetics, 40(5): 663-669, may 2008. ISSN 10614036. doi: 10.1038/ng.142.

18. Kelan Chen, Jiang Hu, Darcy L Moore, Ruijie Liu, Sarah A Kessans, Kelsey Breslin, Isabelle S Lucet, Andrew Keniry, Huei San Leong, Clare L Parish, Douglas J Hilton, Richard J L F Lemmers, Silvère M van der Maarel, Peter E Czabotar, Renwick C J Dobson, Matthew E Ritchie, Graham F Kay, James M Murphy, and Marnie E Blewitt. Genome-wide binding and mechanistic analyses of Smchd1-mediated epigenetic regulation. Proceedings of the National Academy of Sciences, 112(27):E3535-E3544, 2015. ISSN 0027-8424. doi: $10.1073 /$ pnas.1504232112.

19. Josh Quick. One pot native barcoding protocol, loman lab protocols. http://1ab. loman.net/protocols/.

20. Ryan Wick. Porechop. https://github.com/rrwick/Porechop, 2018

21. Heng Li. Minimap2: Pairwise alignment for nucleotide sequences. Bioinformatics, 34(18): 3094-3100, 2018. ISSN 14602059. doi: 10.1093/bioinformatics/bty191.

22. Adam Frankish, Mark Diekhans, Anne-Maud Ferreira, Rory Johnson, Irwin Jungreis, Jane Loveland, Jonathan M. Mudge, Cristina Sisu, James Wright, Joel Armstrong, If Barnes, Andrew Berry, Alexandra Bignell, Silvia Carbonell Sala, Jacqueline Chrast, Fiona Cunningham, Tomás Di Domenico, Sarah Donaldson, lan T. Fiddes, Carlos García Girón, Jose Manuel Gonzalez, Tiago Grego, Matthew Hardy, Thibaut Hourlier, Toby Hunt, Osagie G. Izuogu, Julien Lagarde, Fergal J. Martin, Laura Martínez, Shamika Mohanan, Paul Muir, Fabio C.P. Navarro, Anne Parker, Baikang Pei, Fernando Pozo, Magali Ruffier, Bianca M. Schmitt, Eloise Stapleton, Marie-Marthe Suner, Irina Sycheva, Barbara Uszczynska-Ratajczak, Jinuri Xu, Andrew Yates, Daniel Zerbino, Yan Zhang, Bronwen Aken, Jyoti S. Choudhary, Mark Gerstein, Roderic Guigó, Tim J.P. Hubbard, Manolis Kellis, Benedict Paten, Alexandre Reymond, Michael L. Tress, and Paul Flicek. GENCODE reference annotation for the human and mouse genomes. Nucleic Acids Research, 47(D1):D766-D773, jan 2019. ISSN 03051048. doi: 10.1093/nar/gky955

23. Heng Li, Bob Handsaker, Alec Wysoker, Tim Fennell, Jue Ruan, Nils Homer, Gabor Marth, Goncalo Abecasis, and Richard Durbin. The Sequence Alignment/Map format and SAMtools. Bioinformatics, 25(16):2078-2079, 2009. ISSN 13674803. doi: 10.1093/ bioinformatics/btp352.

24. Yang Liao, Gordon K. Smyth, and Wei Shi. FeatureCounts: An efficient general purpose program for assigning sequence reads to genomic features. Bioinformatics, 30(7):923-930, 2014. ISSN 14602059. doi: 10.1093/bioinformatics/btt656.

25. R Core Team. R: A Language and Environment for Statistical Computing. R Foundation for Statistical Computing, Vienna, Austria, 2019.

26. W. Huber, V. J. Carey, R. Gentleman, S. Anders, M. Carlson, B. S. Carvalho, H. C. Bravo, S. Davis, L. Gatto, T. Girke, R. Gottardo, F. Hahne, K. D. Hansen, R. A. Irizarry, M. Lawrence, M. I. Love, J. MacDonald, V. Obenchain, A. K. Ole's, H. Pag'es, A. Reyes, P. Shannon, G. K. Smyth, D. Tenenbaum, L. Waldron, and M. Morgan. Orchestrating high-throughput genomic analysis with Bioconductor. Nature Methods, 12(2):115-121, 2015.

27. Yang Liao, Gordon K Smyth, and Wei Shi. The Subread aligner: fast, accurate and scalable read mapping by seed-and-vote. Nucleic Acids Research, 41(10):e108, 2013. ISSN 1362 4962 (Electronic). doi: 10.1093/nar/gkt214.

28. Yang Liao, Gordon K Smyth, and Wei Shi. The R package Rsubread is easier, faster, cheaper and better for alignment and quantification of RNA sequencing reads. Nucleic Acids Research, 47(8):e47, 2019. ISSN 0305-1048. doi: 10.1093/nar/gkz114.

29. Bioconductor Core Team. Mus.musculus: Annotation package for the Mus.musculus object, 2015. R package version 1.3.1

30. Mark D. Robinson, Davis J. McCarthy, and Gordon K. Smyth. edgeR: A Bioconductor package for differential expression analysis of digital gene expression data. Bioinformatics, 26 (1):139-140, 2009. ISSN 13674803. doi: 10.1093/bioinformatics/btp616.

31. Davis J. McCarthy, Yunshun Chen, and Gordon K. Smyth. Differential expression analysis of multifactor RNA-Seq experiments with respect to biological variation. Nucleic Acids Research, 40(10):4288-4297, 2012. ISSN 03051048. doi: 10.1093/nar/gks042.

32. Mark D. Robinson and Alicia Oshlack. A scaling normalization method for differential ex pression analysis of RNA-seq data. Genome Biology, 11(3):R25, 2010. ISSN 1465-6906. doi: 10.1186/gb-2010-11-3-r25.

33. Charity W Law, Yunshun Chen, Wei Shi, and Gordon K Smyth. voom: precision weights unlock linear model analysis tools for RNA-seq read counts. Genome Biology, 15(2):R29, 2014. ISSN 1465-6906. doi: 10.1186/gb-2014-15-2-r29.

34. Charity W. Law, Monther Alhamdoosh, Shian Su, Gordon K. Smyth, and Matthew E. Ritchie. RNA-seq analysis is easy as 1-2-3 with limma, Glimma and edgeR. F1000Research, 5(1): 1408, 2016. ISSN 2046-1402. doi: 10.12688/f1000research.9005.2.

35. Ruijie Liu, Aliaksei Z Holik, Shian Su, Natasha Jansz, Kelan Chen, Huei San Leong, Marnie E Blewitt, Marie-Liesse Asselin-Labat, Gordon K Smyth, and Matthew E Ritchie. Why weight? Modelling sample and observational level variability improves power in RNA-seq analyses. Nucleic Acids Research, 43(15):e97, 2015. ISSN 0305-1048. doi: 10.1093/nar/gkv412.

36. Gordon K Smyth. Linear Models and Empirical Bayes Methods for Assessing Differential Expression in Microarray Experiments. Statistical Applications in Genetics and Molecular Biology, 3:Article 3, 2004. ISSN 1544-6115. doi: 10.2202/1544-6115.1027.

37. Yoav Benjamini and Yosef Hochberg. Controlling The False Discovery Rate - A Practical
And Powerful Approach To Multiple Testing. Journal of the Royal Statistical Society Series B, 57(1):289-300, 1995. ISSN 00359246. doi: 10.2307/2346101.

38. Ruijie Liu, Kelan Chen, Natasha Jansz, Marnie E Blewitt, and Matthew E Ritchie. Transcriptional profiling of the epigenetic regulator Smchd1. Genomics Data, 7:144-147, 2016. ISSN 22135960. doi: 10.1016/j.gdata.2015.12.027.

39. Di Wu, Elgene Lim, François Vaillant, Marie Liesse Asselin-Labat, Jane E. Visvader, and Gordon K. Smyth. ROAST: Rotation gene set tests for complex microarray experiments. Bioinformatics, 26(17):2176-2182, 2010. ISSN 13674803. doi: 10.1093/bioinformatics/ btq 401 .

40. Dana Wyman and Ali Mortazavi. TranscriptClean: Variant-aware correction of indels, mismatches and splice junctions in long-read transcripts. Bioinformatics, 35(2):340-342, 2019. ISSN 14602059. doi: 10.1093/bioinformatics/bty483.

41. Manuel Tardaguila, Lorena De La Fuente, Cristina Marti, Cécile Pereira, Francisco Jose Pardo-Palacios, Hector Del Risco, Marc Ferrell, Maravillas Mellado, Marissa Macchietto, Kenneth Verheggen, Mariola Edelmann, lakes Ezkurdia, Jesus Vazquez, Michael Tress, Ali Mortazavi, Lennart Martens, Susana Rodriguez-Navarro, Victoria Moreno-Manzano, and Ana Conesa. SQANTI: Extensive characterization of long-read transcript sequences for quality control in full-length transcriptome identification and quantification. Genome Research, 28(3):396-411, 2018. ISSN 15495469. doi: 10.1101/gr.222976.117.

42. Alicia Oshlack and Matthew J. Wakefield. Transcript length bias in RNA-seq data confounds systems biology. Biology Direct, 4:14, 2009. ISSN 17456150. doi: 10.1186/1745-6150-4-14. 
bioRxiv preprint doi: https://doi.org/10.1101/2020.06.28.176727; this version posted June 29, 2020. The copyright holder for this preprint (which was not certified by peer review) is the author/funder, who has granted bioRxiv a license to display the preprint in perpetuity. It is made available under aCC-BY-ND 4.0 International license.

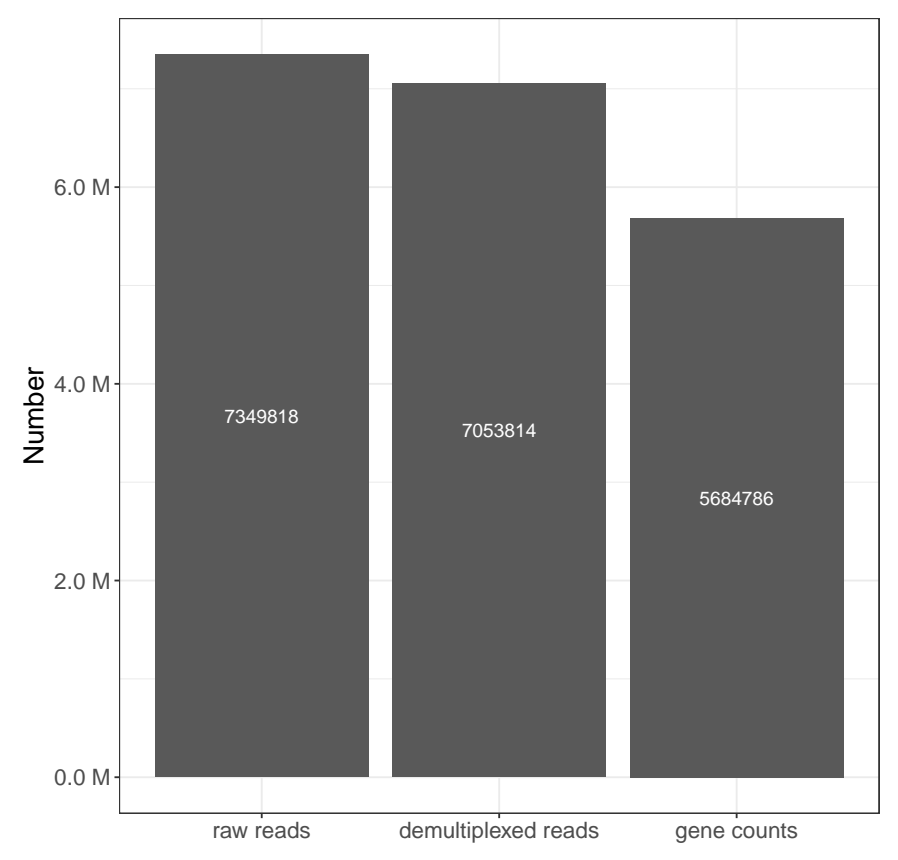

Fig. S1. The number of pass (average base quality score >7) raw reads, trimmed and demultiplexed reads and assigned reads in the sequins dataset.

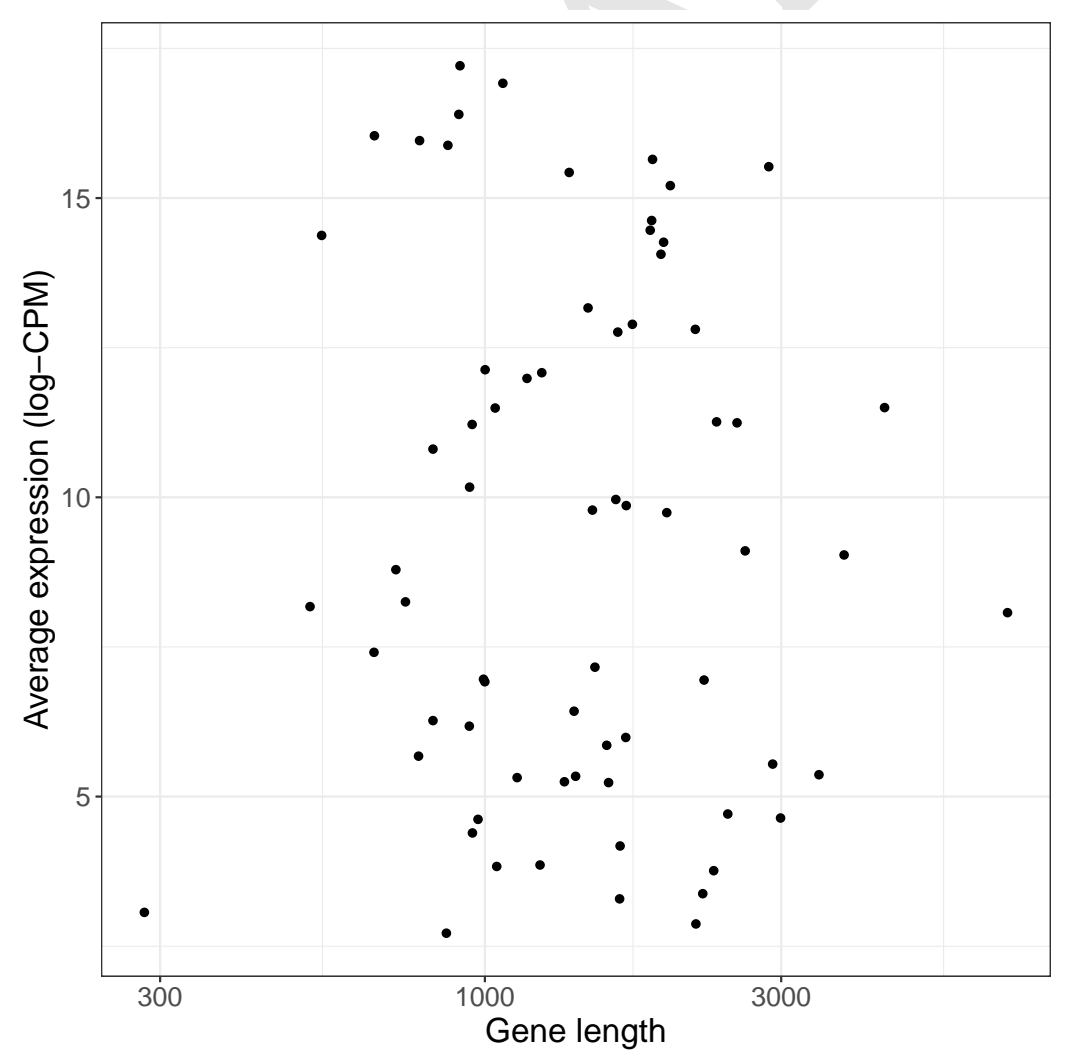

Fig. S2. Correlation between gene length and average gene expression (log-CPM) in the sequins dataset. 
voom: Mean-variance trend

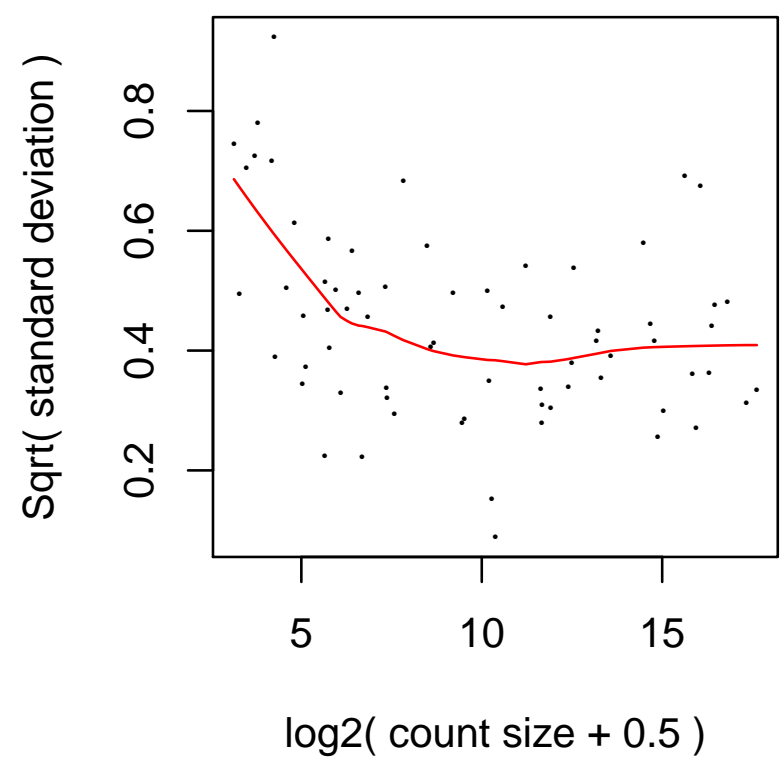

Sample-specific weights

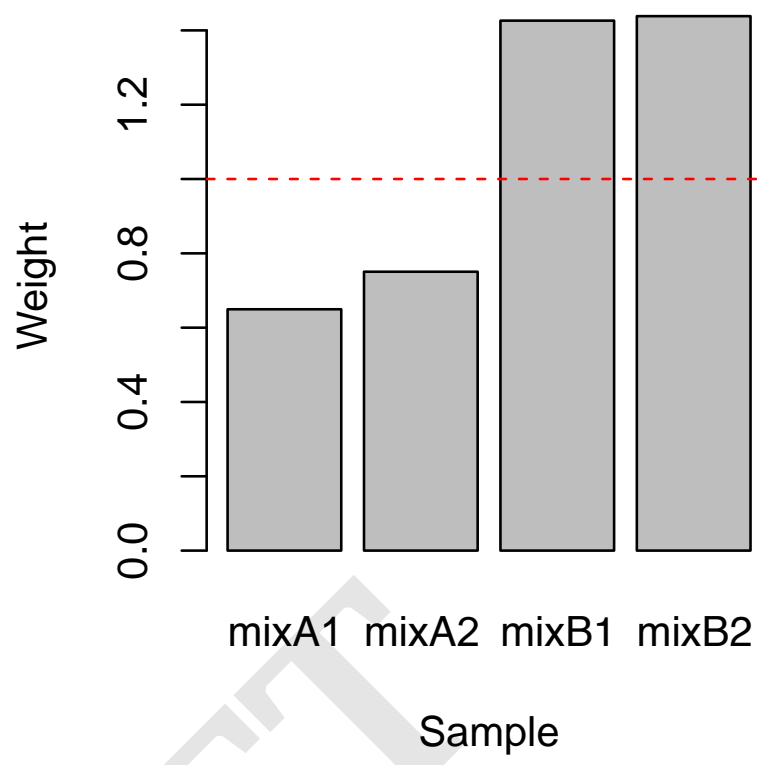

Fig. S3. Voom mean-variance trend in the sequins data where points represent genes, and sample-specific weights obtained from the voomWithQualityWeights function.

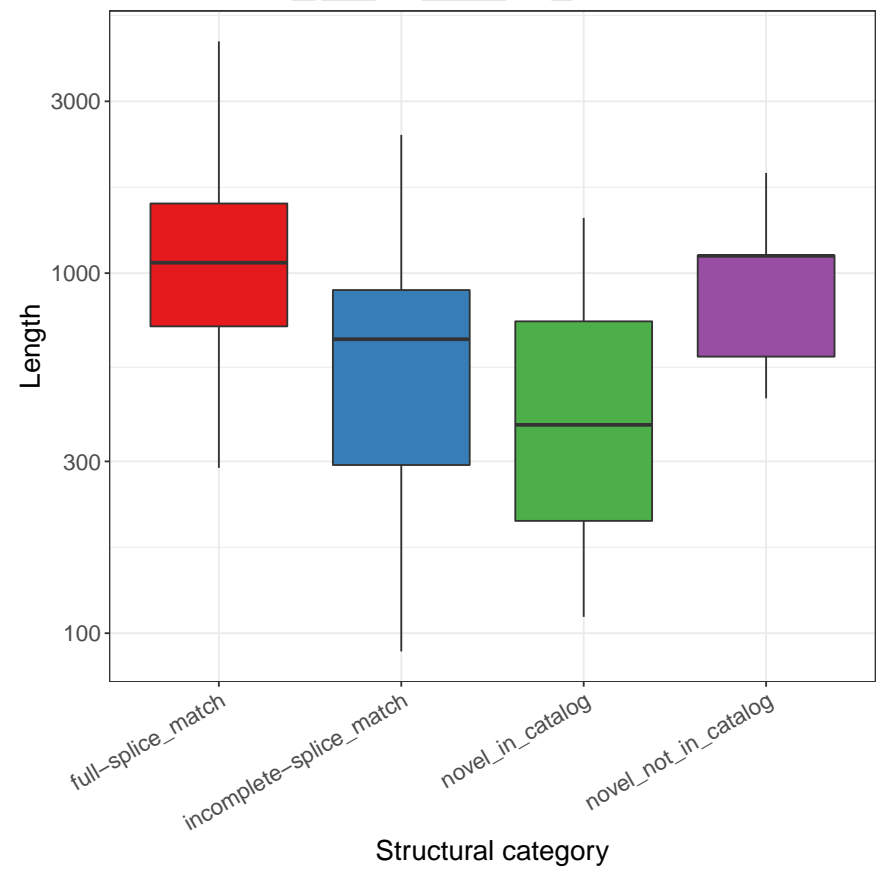

Fig. S4. A box plot showing the length distribution of isoforms identified by FLAMES in the sequins dataset, stratified by isoform structural categories. 
bioRxiv preprint doi: https://doi.org/10.1101/2020.06.28.176727; this version posted June 29, 2020. The copyright holder for this preprint (which was not certified by peer review) is the author/funder, who has granted bioRxiv a license to display the preprint in perpetuity. It is made available under aCC-BY-ND 4.0 International license.

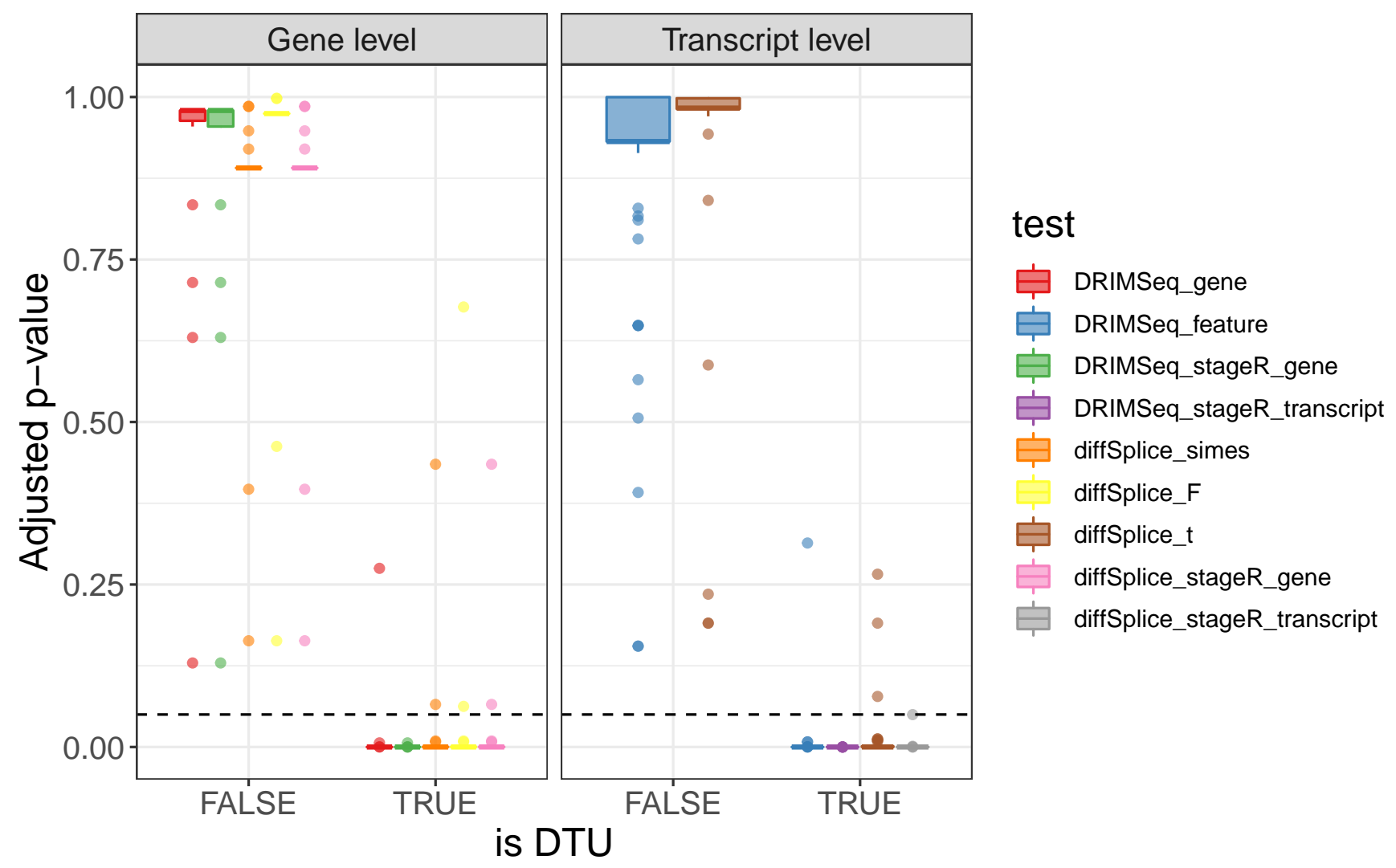

Fig. S5. A box plot showing the distribution of adjusted $p$-values from different tests of DTU for the sequins data, faceted by whether the test is performed at the gene-level or transcript-level and stratified by whether the gene or transcript has true DTU. The dashed horizontal line shows the adjusted $p$-value cutoff of 0.05 .

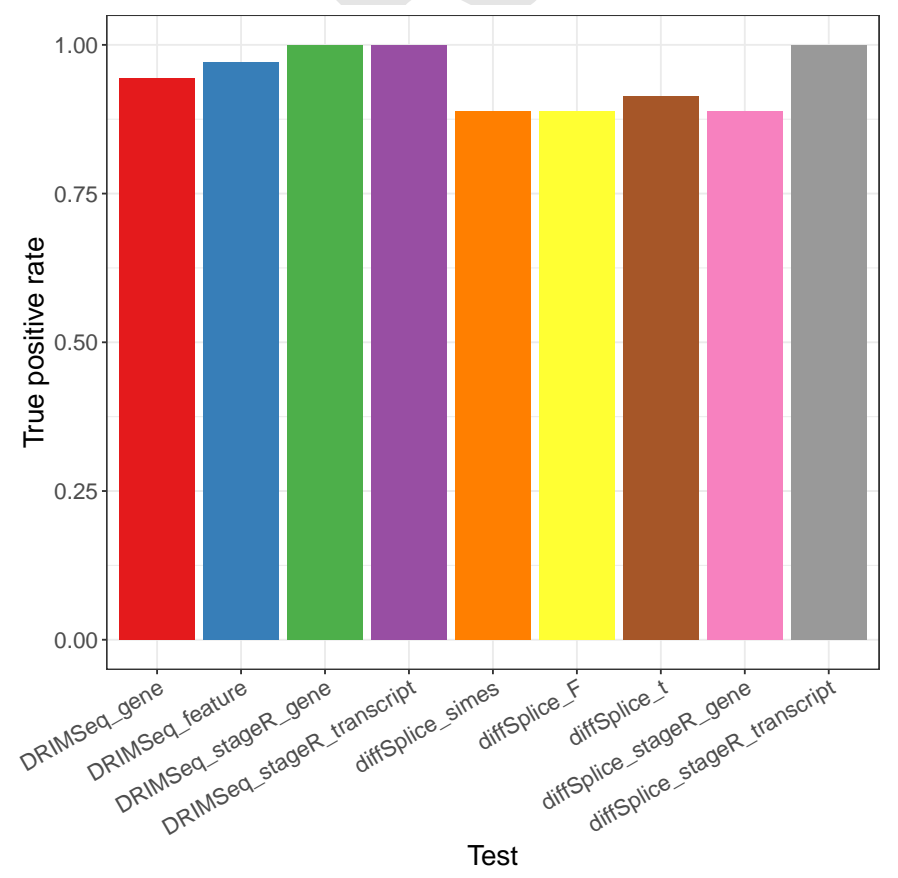

Fig. S6. A bar plot showing the true positive rate (TPR) from different tests of DTU for the sequins data. 
bioRxiv preprint doi: https://doi.org/10.1101/2020.06.28.176727; this version posted June 29, 2020. The copyright holder for this preprint (which was not certified by peer review) is the author/funder, who has granted bioRxiv a license to display the preprint in perpetuity. It is made available under aCC-BY-ND 4.0 International license.

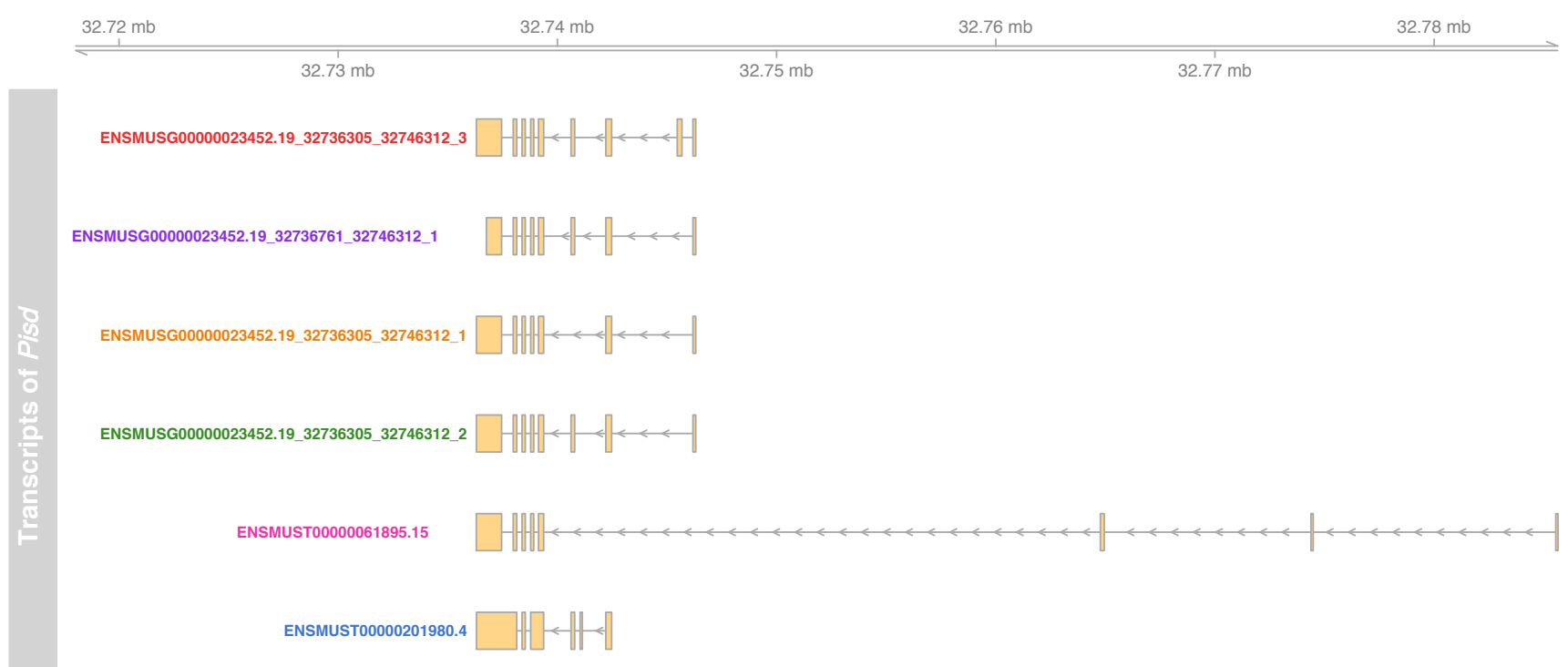

Fig. S7. Different isoforms of gene Pisd in the NSC dataset identified by FLAMES.

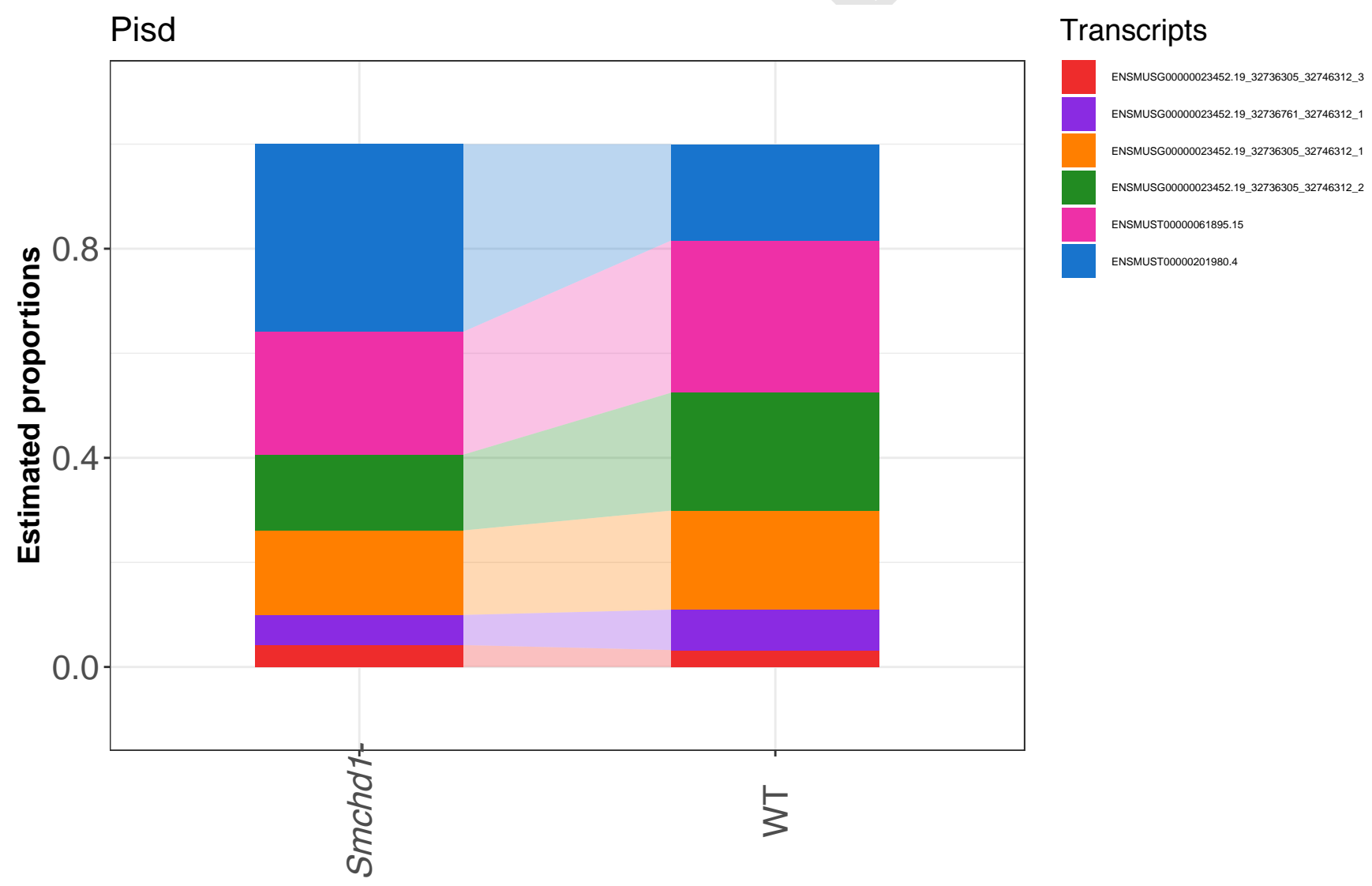

Groups

Fig. S8. A ribbon plot showing the estimated isoform proportions for the gene Pisd in the NSC dataset. 